\title{
First Time Demonstration of the Quantum Interference Effect during Integration of Cognition and Emotion in Children
}

\author{
Elio Conte1,2, Rui Freire Lucas ${ }^{3,4}$ \\ ${ }^{1}$ School of Advanced International Studies on Applied Theoretical and Non Linear Methodologies of Physics, \\ Bari, Italy \\ ${ }^{2}$ Department of Basic Sciences, Neuroscience and Sense Organs, University Aldo Moro, Bari, Italy \\ ${ }^{3}$ University Hospital Centre of Vaud, Department of Psychiatry, Vaud, Switzerland \\ ${ }^{4}$ Centre for Philosophy of Sciences of the University of Lisbon, Lisbon, Portugal \\ Email: elio.conte@fatswebnet.it
}

Received 11 March 2015; accepted 25 April 2015; published 30 April 2015

Copyright (C) 2015 by authors and Scientific Research Publishing Inc.

This work is licensed under the Creative Commons Attribution International License (CC BY).

http://creativecommons.org/licenses/by/4.0/

c) (i) Open Access

\begin{abstract}
Quantum cognition is a scientific approach to cognitive phenomena which makes use of the mathematical formalism of quantum theory. Quantum interference effect constitutes one of this theory's main tenets and has been repeatedly demonstrated experimentally, in the last decade, in adult subjects. In the present paper, we aim to demonstrate, for the first time, the existence of the quantum interference effect on children during an experiment involving an integration of cognition and emotion. Our positive results consolidate the presuppositions of quantum cognition, enlarging its field of application to children's mental apparatus and evidence the important question to consider the quantum model in the current investigated question of the interaction of cognition and emotion in children at neurological and psychological levels.
\end{abstract}

\section{Keywords}

Quantum Cognition, Quantum Interference Effect, Quantum Mechanics, Emotion-Cognition Interaction in Children

\section{Introduction}

Cognition is the mental process of knowing, including aspects such as awareness, perception, reasoning, and judgment. It relates what comes to be known, perception, reasoning or intuition, and knowledge. One of our 
long-range goals is to understand the neural underpinnings of human emotion and to examine how cognitive and emotional brain systems interact in the generation of complex behaviour. Cognitive science is already an advanced discipline, but we are still far from a thorough understanding on cognitive mechanisms and on the relationship between cognition and emotion.

In the last years, many studies have greatly contributed to the development of a new scientific approach presently called quantum cognition. Starting in 2003, A. Y. Khrennikov first studied this field in detail; from 2003 onwards, in collaboration with one of us (EC) (see Conte [1] for details on his initial contributions already in 1980-1986), these two authors developed theoretical and experimental results contributing to the advance in this field. For a comprehensive review, one may read Khrennikov [2] and Conte [1]. Other authors have given important contributions to this matter as well (Aerts, 1991 [3]; Bruza and Kitto, 2007, Busemeyer (see Busemeyer and Bruza, 2012) [4] and still Snyder [5] and Bourdley [6] [7]).

All these investigations have given us proofs of quantum cognition's validity in the study of human cognition. Specifically, our experiments have granted us with decisive evidence regarding the existence of quantum interference during perceptive and cognitive performances in humans [1] [8]-[10].

For the purpose of the present paper, we highlight our recent experiments, with adult subjects, where we have used ambiguous figures and the Stroop effect to test semantic conflicts, and cognitive anomalies such as the conjunction fallacy (see Conte et al. [11]; Conte [1] [9] and references therein for a complete list of the papers). At an ontological level, Conte's main conclusions go against some of our most solid convictions. Currently, our historical position in physical science, as well as in psychology and neurosciences, posits matter in space and time, existing as an objective entity independent of human mental entities. These entities of course operate in the abstract, in a condition of total separation and independence, and one of constant interaction with external objective material reality. The mechanism is as follows: we have interaction matter-mental reality. An object is perceived from the brain by the sense organs. They transmit information and the perceived features are transformed by the sense organs into neural activities by an operation that we call transduction. Consequently, brain realizes a mental representation of the object. This is a well established stimulus-response like mechanism that of course at a macroscopic scale may be experimentally verified each time, and we have no need to debate about it. However, this model still contains a peculiar feature: both matter and mental entities are well separated, matter is positioned in space and in time while mental entities remain confined in an abstract space that we are unable to characterize in detail. It remains a difficult matter to establish if and where they are actually located. In recent years, some Conte's studies have been developed on the basis of a quantum model. It suggests a different perspective, indicating the possibility that actually human cognitive dynamics arises, coexisting $a b$ initio in strong relation with the material sphere. This has indicated us that the conceptual foundations of quantum mechanics can have an elective role in understanding the way through which primary elements of cognition on one hand and material reality on the other are able to coexist $a b$ initio. As previously outlined, we are accustomed to considering reality as affected by a definite dualism. On one side, we admit that matter exists per se independently from our observation and measurement (particles, atomic nuclei, molecules...). On the other side, we admit the existence of a human cognitive structure and function, which is able to receive inputs from the outside sphere, and to elaborate such information by performing semantic acts and decisions. Now, Conte's studies on quantum mechanics have led us to an opposite direction. This is to say that they have given us evidence that quantum mechanics has some basic and peculiar features whose origin is located in logic and cognition [12]-[14]. It contains an indication of ab initio existing of our primary cognitive elements [12] [15] [16]. In particular, the wave function of quantum mechanics, which is the pillar of the theory, must be considered as a factor of knowledge closely related with cognitive performance. For details, see Conte [10] [17]-[19].

As an overall conclusion, it arises that there are stages of our reality in which it is impossible to unconditionally define the truth. Mental entities are algebraically represented in the quantum model by abstract elements of a so-called Clifford algebra whose elements, when characterizing logic statements, assume a truth content that is relative to the transformation we are considering. The truth is subjected to an irreducible intrinsic and ontological indetermination as well as the same concept of existence [14] [16] [20].

Therefore, the first step is that at this level we lose the possibility to unconditionally define the truth. In detail, at these levels of reality, the fundamental features of cognition (and thus of the logic) and language-i.e. conceptual entities - acquire the same importance of what is being described. Here, we cannot separate the features of matter per se from the features of cognition and language used to describe it. Conceptual entities are inseparable from matter as object of our cognitive performance. They coexist. 
This conclusion, shared also previously by other authors such as Orlov [20], nevertheless seems to subvert our traditional approach to cognitive science, opening new and interesting perspectives in this field. The delineated quantum model is explained in detail in several Conte's papers, but, in particular, some important features of his quantum model and of further importance in neuroscience are discussed in detail in the section entitled "Application of the Present Theory to Cognitive Processes in Psychology" of the paper given in [21]. Therefore, since it is important to elaborate all the features of the assumed quantum model, we consider that the previously mentioned section is mainly an integrating part of the present paper. Consequently, we retain this mentioned section as basic neuroscience foundation of the present paper, in addition to the other papers mentioned in references.

We consider the present study as a further attempt to support the quantum cognitive model that in our opinion is of valuable interest in psychology as well as in neurosciences. On the other hand, in order to support such evidences, we need new experimental confirmations. That's exactly the aim of the present paper.

\section{The Basic Theoretical Elaboration}

The adopted quantum model is rather simple. Let us recall one basic postulate of the quantum theory. Any physical system is described by a state vector $\psi$ which is positioned in a Hilbert space, a complex vector space equipped with an inner product $\langle\varphi \mid \psi\rangle$. A state vector has unit norm $\langle\psi \mid \psi\rangle=1$.

One of the basic tenets of quantum theory is the so called Superposition Principle. It states that any two state vectors (in a Hilbert space) of a quantum mechanical system, can be coherently and linearly added together to form another valid state of the system: For $\varphi, \psi \in H$, we have, $|\Psi\rangle=a|\psi\rangle+b|\varphi\rangle$; with $a, b \in C ;|a|^{2}$ being the probability for state $\psi ;|b|^{2}$ being the probability for state $\varphi$; and $\psi$ and $\varphi$ forming an orthonormal basis. Out of the mathematical rigour, the importance of the superposition principle resides in the innovative profile that it delineates about quantum reality. This principle sabotages our traditional approach to reality. This happens because it admits the existence of multiple states simultaneously which is the same as considering that the reality outlined by quantum mechanics does not have a direct counterpart in the Boolean logic. In our current everyday experience, at a macroscopic level, we are accustomed, for example, to observe a traffic light that is always either red either green. The Superposition Principle admits instead that alternatives coexist simultaneously which, in the given example, is the same as saying that both red and green lights coexist simultaneously (at least on the level of ontological potentiality). In brief, the Superposition Principle responds to the "And" rather to the "Or" logic. While it seems rather difficult to conceive this principle on the macroscopic material level, we are nevertheless able to apply it on a cognitive level, admitting the possibility of simultaneous coexistence of two mental states in humans. We have a large set of possible empirical evidences at psychological level.

There is still another feature that results of great importance in Superposition Principle. As seen previously, we represent the state as $|\Psi\rangle=a|\psi\rangle+b|\varphi\rangle$; with $a, b \in C ;|a|^{2}$ being the probability for state $\psi$; $|b|^{2}$ being the probability for state $\varphi$; and $\psi$ and $\varphi$ forming an orthonormal basis.

The presence of complex numbers $a, b \in C$ indicates probability amplitudes so that $|a|^{2}$ being the probability for state $\psi ;|b|^{2}$ being the probability for state $\varphi$. Consequently, the emerging model of reality as described from quantum mechanics is that one of a structure that in principle is marked from an intrinsic and irreducible indetermination in which the probability field is responsible of the events that we observe. Probability in quantum mechanics no more relates our ignorance about the set of possible happening events; it marks the irreducible indetermination of our reality at quantum level.

We shall now examine the problem of the quantum interference which is strongly related with the Superposition Principle in quantum mechanics.

Let us consider that a device is spraying particles at a screen in which there are two opening slits which may or may not be covered.

First, suppose the experiment is carried out with only one slit opened, say Slit 1 . The particles target the detecting screen and the experimental outcome is represented by a curve $P_{1}(x)$ in a way that $P_{1}(x) \delta(x)$ represents the probability of a particle landing in the range $(x, x+\delta x)$. If we cover this slit and open the other, we will obtain a curve $P_{2}(x)$ similar to $P_{1}(x)$ according to the following picture (Figure 1).

This is of course exactly what we expect when performing such experiment at a macroscopic level. Finally, we admit that both slits are opened. We would then observe that the particles would sometimes come through Slit 1 and sometimes through Slit 2, varying between the two possibilities in a random way. This will produce 


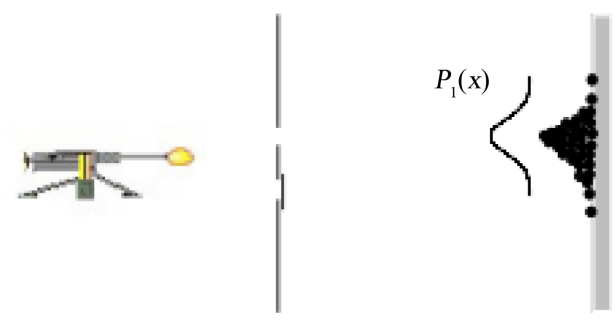

(a)
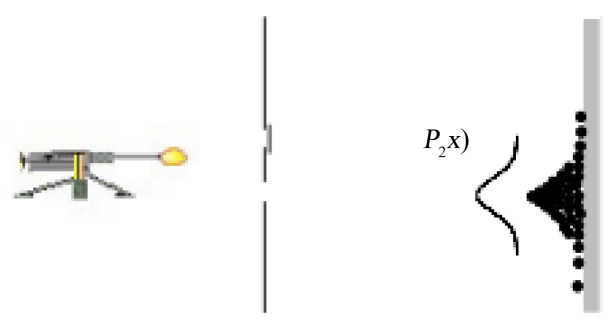

(b)

Figure 1. Edge-on view of particle source, two-slit diaphragm, and detection screen with respective patterns for situation A (top-slit open; bottom-slit closed) and situation B (top-slit closed; bottom-slit open).

two piles behind each slit which constitute the sum of the results that would be observed with one or the other slit opened.

Consequently, we should have

$$
P_{12}(x)=P_{1}(x)+P_{2}(x)
$$

Instead, according to quantum mechanics we have

$$
P_{12}(x)=P_{1}(x)+P_{2}(x)+2 \sqrt{P_{1}(x) P_{2}(x)} \cos \vartheta \neq P_{1}(x)+P_{2}(x)
$$

We obtain what it is called a typical quantum interference pattern where a new term is given by

$$
2 \sqrt{P_{1}(x) P_{2}(x)} \cos \vartheta .
$$

In conclusion, quantum mechanics introduces a model of reality that is marked by an intrinsic, irreducible indetermination signed from probability amplitudes and from the abstract field of probabilities. As previously indicated, a detailed explanation of such question with particular relation to neuroscience is given in [20] [22]-[26].

In addition we have still an important feature to be outlined. As said, quantum interference patterns represent a further distinctive peculiarity of quantum theory.

We have to evidence a very remarkable feature: in accord with the previous studies of Orlov, we have found basic theoretical results evidencing that the previously mentioned quantum interference arises also when we use logic statements. We have given particular evidence of such result in our papers [1] [13]. Therefore, in order to obtain quantum interference we do not need particles targeting the detecting screen as previously discussed. We obtain the same quantum interference using mental entities as abstract logic statements. This is an important result on the way to support our quantum model as previously detailed and to evidence the particular role of quantum mechanics in the dynamics of mental entities. Soon after we have to add still some comment about the manner in which we have used here such term of probability. Probability filed is still an abstract entity having, however, a specific role. Margenau [22] as well as Eccles [23] [24], discussed the essence and the intrinsic role of such probability fields in the dynamics of our mental events. In this approach probabilities represent an abstract field that of course is responsible of events in quantum dynamical reality. Mental events cause neural events analogously to the manner in which probability fields of quantum mechanics are causatively responsible for physical events (Wolf [25]). Beck and Eccles [26] formulated a quantum approach entering quantum mechanics in brain dynamics in connection with exocytosis that, as it is well known, relates the release of the contents of a vesicle, filled with neurotransmitter, from a nerve terminal into a synaptic cleft. In order to describe such basic process, they used a pillar of quantum mechanics, the quantum mechanical tunnelling. Also Wolker obtained similar results [27] and also Conte [28].

On the basis of such results, we introduce the following statement, as a hypothesis to be tested experimentally: in decision-making, every time a subject is called to make a decision about a posed task and such task is marked by an intrinsic ambiguity, that is to say by inner and irreducible indetermination, the Superposition Principle of quantum mechanics holds and consequently we must expect quantum interference. Obviously, such basic statement must have a neurological counterpart that requires to be studied in detail. Briefly we may outline it here in the following manner: brain not only delineates consciousness awareness "to see", it also "expects" probabilistic predictive scenarios based on its innate tendency to the foresight of the future. Such probabilistic brain feature to do conscious predictions does not relate rules of classical probability but instead quantum probabilistic 
rules as obtained and explained in detail by us in our previous papers [17] [18].

Finally, the hypothesis of existing quantum interference in mental processes not only has been evidenced by us using basic theoretical elaborations, but also has already been tested by us experimentally through the use of ambiguous figures and the Stroop test, known for its capacity to induce semantic conflicts. We have yet made further experiments in the context of cognitive anomalies such as the conjunction fallacy. The aim of the present research was to perform an experiment that has basic analogies with an experiment that was previously evidenced from other authors (Busemeyer and Wang [29]; Busemeyer, Wang and Lambert-Mogiliansky [30]). The finality of our experiment, however, is different.

Let us fix it.

It is of neurological and psychological interest to investigate the integration of the emotion and of cognition in children. As it is obvious it is constituting presently the object of crescent interest (Calkins et al. [31]). The aim of our experiment was to perform it in children in order to investigate if quantum interference arises and thus if the role of quantum mechanics and of the quantum model previously discussed, must be accounted during specialized neurological as well as psychological investigation on integration of emotion and cognition during the development in children.

\section{The Experiment}

Forty healthy children with ages between 6 and 11 years (mean age $8 \pm 1.7$ ) were randomly recruited in a commercial area, after voluntary acceptance to participate in the experiment. and written signature of parents agreement. They were then randomly allocated into two subgroups-each composed of 10 subjects of each sex-identified as groups A and B. The first group was shown a picture displayed on a tablet screen (Figure 2), and then asked if they felt more prompt to attack the represented element or to withdraw from it. We've shown the same picture to group B however, before submitting them to the precedent task, we've asked them to first identify the evoked feelings through the question "does this picture makes you angry or scared?". In this manner, in such group B we objectively marked more integration of emotion and cognition respect to group A.

With this experiment, it was our intention to demonstrate a quantum interference effect. Therefore, we've introduced, in Group A, a dichotomous variable $A$, taking the possible two values, +1 or -1 . The probabilities $p(A=+1)$ and $p(A=-1)$ were then estimated. To Group B we've introduced a new variable $B$, with possible values $B=+1$ or $B=-1$, before submitting them to the task already proposed to Group A. In this case the following probabilities were estimated $p(B=+1), \quad p(B=-1), \quad p(A=+1 / B=+1), \quad p(A=+1 / B=-1)$, $p(A=-1 / B=+1)$, and $p(A=-1 / B=-1)$.

Note the experimental situation: if, during the perception and cognition effort of the subjects submitted to the tasks, quantum mechanics did not apply, classical Bayes's theorem should hold and we should obtain, $p(A=+1)$ (Group A) $=p(B=+1) p(A=+1 / B=+1)+p(B=-1) p(A=+1 / B=-1) \quad$ (Group B) and a similarly expression for $p(A=-1)$.

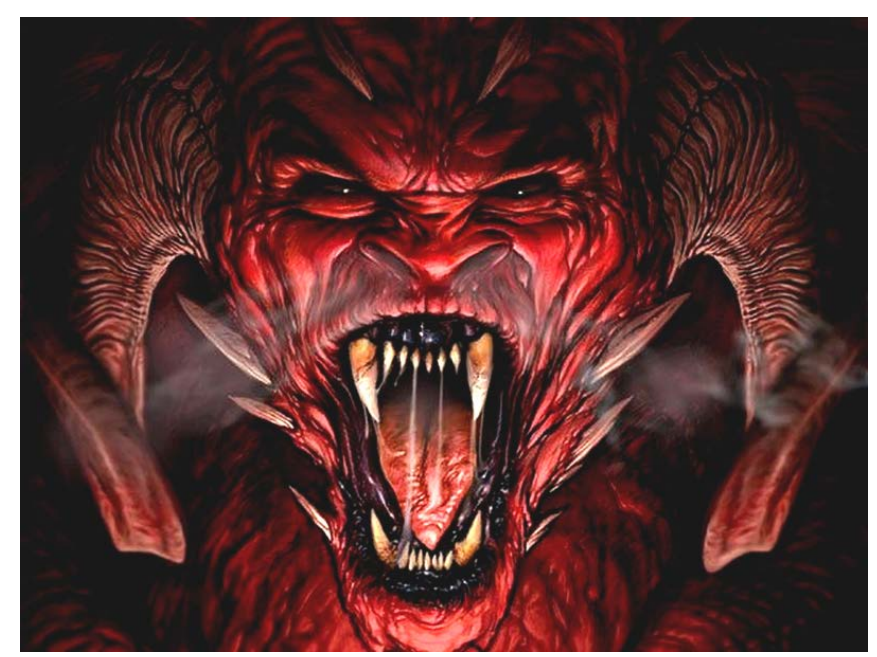

Figure 2. Presented picture. 
If instead the superposition principle and quantum interference were to be manifested in this experiment-thus confirming a role for quantum mechanics during the perception and the cognition of children, we should have instead,

$$
\begin{aligned}
p(A=+1)= & p(B=+1) p(A=+1 / B=+1)+p(B=-1) p(A=+1 / B=-1) \\
& +2 \sqrt{p(B=+1) p(A=+1 / B=+1) p(A=-1 / B=+1)} \cos \vartheta
\end{aligned}
$$

Implying the presence of the quantum interference term

$$
2 \sqrt{p(B=+1) p(A=+1 / B=+1) p(A=-1 / B=+1)} \cos \vartheta
$$

\section{Results and Conclusions}

We obtained the following results.

\begin{tabular}{ccc}
\hline Group A & Group B & \\
\hline Variable A & Variable B & Variable B/A \\
\hline$P(+1)=0.15$ & $P(+1)=2 / 20=0.1$ & $P(A=+1 / B=+1)=0.5, P(A=+1 / B=-1)=0.167$ \\
$P(-1)=0.85$ & $P(-1)=0.9$ & $P(A=-1 / B=+1)=0.5, P(A=-1 / B=-1)=0.833$ \\
\hline
\end{tabular}

Using the formula previously outlined, we obtained for $p(A=+1)$ a value $\cos \vartheta_{+}=-0.29011$ and $\vartheta_{+}=1.865146$.

Generally speaking, we may therefore conclude that children's perceptive-cognitive performance is subject to quantum interference. This conclusion, already achieved with adult subjects, strengthens the role of quantum cognition in the study of human cognitive operations, eventually leading us to the development of a more complete grounded theory of the mind which can help better understand not only human personality, but also mental disorders.

Specifically, these results reveal themselves to be particularly meaningful in the way that they point us to untraveled directions. As said, neurologists, in particular developmental theorists from some time, are elaborating that emotion and cognition are inseparable components of the developmental process in children. Some results evidence that the two components are fully integrated from different factors and in particular school age. However, although valuable theoretical work describing this interaction and empirical work have been conducted on this matter, final evidence has not still been obtained and a rather detailed model able to quantify the integration is still missing. The aim of the studies is to address the co-development of emotional and cognitive processes by integrating theoretical and empirical work on these processes. We retain to have reached a preliminary but important result in this paper, since we have formulated a detailed quantum model whose details of course may be further deepened as illustrated in Conte [10]; we have reached evidence of such existing integration emotion-cognition attributing to the variable $\mathrm{B}$ more the value of a pure emotive variable and to A that one of a more pure cognitive variable, and finally, for the first time, we have been able to quantify the entity of the integration by evaluating the interference term whose value has been previously reported. This methodological procedure opens the way to study more and more detailed experimental conditions in which an accurate analysis of the integration emotion-cognition is required not only for basic theoretical explanations but also for correlated clinical evaluations. A final comment: neuroscience needs to find a direct link with our quantum model. All the basic features of our quantum algebraic model linked to neuroscience are reported in the literature. In particular, we suggest reading the paper quoted in [21], and in detail the section entitled "Application of the Present Theory to Cognitive Processes in Psychology".

\section{References}

[1] Conte, E. (2011) Advances in Application of Quantum Mechanics in Neuroscience and Psychology: A Clifford Algebraic Approach. Nova Science Publishers, New York.

[2] Khrennikov, A. (2010) Ubiquitous Quantum Structure: From Psychology to Finances. Springer, Berlin.

[3] Aerts, D. (1991) A Mechanistic Classical Laboratory Situation Violating the Bell Inequalities with $2 ・ \sqrt{ } 2$, Exactly "in 
the Same Way" as Its Violations by the EPR Experiments. Helvetica Physica Acta, 64, 1-23.

[4] Busemeyer, J.R. and Bruza, P.D. (2012) Quantum Models of Cognition and Decisions. Cambridge University Press, Cambridge. http://dx.doi.org/10.1017/CBO9780511997716

[5] Snyder, M.D. (1995) On the Quantum Mechanical Wave Function as a Link between Cognition and the Physical World: A Role for Psychology. http://cogprints.org/2196/

[6] Bordley, R.F. (1983) Modeling Quantum Behavior with Standard (Nonquantum) Probability Theory. Journal of Mathematical Physics, 24, 2411-2421. http://dx.doi.org/10.1063/1.525622

[7] Bordley, R.F. (1997) Experiment-Dependent Probabilities in Quantum-Mechanics and Psychology. Physics Essays, 10, 401-406.

[8] Conte, E., Todarello, O., Federici, A., Vitiello, F., Lopane, M., Khrennikov, A.Y. and Zbilut, J.P. (2007) Some Remarks on an Experiment Suggesting Quantum-Like Behavior of Cognitive Entities and Formulation of an Abstract Quantum Mechanical Formalism to Describe Cognitive Entity and Its Dynamics. Chaos, Solitions and Fractals, 31, 1076-1088. http://dx.doi.org/10.1016/j.chaos.2005.09.061

[9] Conte, E., Khrennikov, A.Y., Todarello, O., Federici, A., Mendolicchio, L. and Zbilut, J.P. (2009) Mental States Follow Quantum Mechanics during Perception and Cognition of Ambiguous Figures. Journal of Open Systems and Information Dynamics, 16, 1-17.

[10] Conte, E., Santacroce, N., Laterza, V., Conte, S., Federici, A. and Todarello, O. (2012) The Brain Knows More than It Admits: A Quantum Model and Its Experimental Confirmation. Electronic Journal of Theoretical Physics, 9, 72-110.

[11] Conte, E., Khrennikov, A.Y., Todarello, O., De Robertis, R., Federici, A. and Zbilut, J.P. (2011) On the Possibility That We Think in a Quantum Mechanical Manner: An Experimental Verification of Existing Quantum Interference Effects in Cognitive Anomaly of Conjunction Fallacy. Chaos and Complexity Letters, 4, 123-136.

[12] Conte, E. (2011) On the Logical Origins of Quantum Mechanics Demonstrated by Using Clifford Algebra: A Proof That Quantum Interference Arises in a Clifford Algebraic Formulation of Quantum Mechanics. Electronic Journal of Theoretical Physics, 8, 109-126.

[13] Conte, E. (2011) On the Logical Origins of Quantum Mechanics Demonstrated by Using Clifford Algebra. NeuroQuantology, 9, 231-242. http://dx.doi.org/10.14704/nq.2011.9.2.397

[14] Conte, E. (2014) Can Current Quantum Cognition Studies Give Indication on the Manner in Which Human Cognition Arose Ab Initio? Psychology, 5, 798-800. http://dx.doi.org/10.4236/psych.2014.58090

[15] Conte, E. (2013) Are Information, Cognition, and the Principle of Existence Intrinsically Structured in the Quantum Model of Reality? Advanced Studies in Theoretical Physics, 7, 797-818.

[16] Conte, E. (2013) A Clifford Algebraic Analysis Gives Mathematical Explanation of Quantization of Quantum Theory and Delineates a Model of Quantum Reality in Which Information, Primitive Cognition Entities and a Principle of Existence Are Intrinsically Represented Ab Initio. World Journal of Neuroscience, 3, 157-170. http://dx.doi.org/10.4236/wjns.2013.33021

[17] Conte, E. (2014) Answer to Giancarlo Ghirardi: Quantum Superpositions and Definite Perceptions: Envisaging New Feasible Experimental Tests. A Novel Proposal for Quantum Mechanics, Perception and Cognitive Science? International Journal of Theoretical Physics, 54, 672-679. http://dx.doi.org/10.1007/s10773-014-2259-6

[18] Conte, E. (2015) Additional Comments Added to Our Recent Answer to G. Ghirardi. Journal of Modern Physics, 6, 12-15. http://dx.doi.org/10.4236/jmp.2015.61002

[19] Conte E. (2015) What Path Monitor: A Brief Note on Quantum Cognition and Quantum Interference, the Role of the Knowledge Factor. Psychology, 6, 291-296. http://dx.doi.org/10.4236/psych.2015.63029

[20] Orlov, Y. (1996) Peculiarities of Quantum Mechanics: Origins and Meaning. http://arxiv.org/abs/quant-ph/9607017

[21] Conte, E., Altamura, M., De Salvia, M., Federici, A., Bellomo, A. and Zbilut, J.P. (2011) On a New Form of Chaos Based on Deterministic Chaos plus Added Quantum Mechanical Components: The Perspectives of Application in Cognitive Process in Psychology. Chaos and Complexity Letters, 5, 27-59.

[22] Margenau, H. (1984) The Miracle of Existence. Ox Bow Press, Woodbridge.

[23] Eccles, J.C. (1990) A Unitary Hypothesis of Mind-Brain Interaction in the Cerebral Cortex. Proceedings of the Royal Society B, 240, 433-451. http://dx.doi.org/10.1098/rspb.1990.0047

[24] Eccles, J.C. (1994) How the Self Controls Its Brain. Springer, Berlin, Heidelberg and New York. http://dx.doi.org/10.1007/978-3-642-49224-2

[25] Wolf, F.A. (1989) On the Quantum Physical Theory of Subjective Antedating. Journal of Theoretical Biology, 136, 1319. http://dx.doi.org/10.1016/S0022-5193(89)80185-7

[26] Beck, F. and Eccles, J.C. (1994) Quantum Processes in the Brain: A Scientific Basis of Consciousness. Cognitive Studies, 5, 95-109. 
[27] Walker, E.H. (1977) Quantum Mechanical Tunneling in Synaptic and Ephaptic Transmission. International Journal of Quantum Chemistry, 11, 103-127. http://dx.doi.org/10.1002/qua.560110109

[28] Conte, E. (2010) On the Possibility That We Think in a Quantum Probabilistic Manner. NeuroQuantology, 8, 3-47. http://dx.doi.org/10.14704/nq.2010.8.4.349

[29] Busemeyer, J.R. and Wang, Z. (2007) Quantum Information Processing Explanation for Interactions between Inferences and Decisions. Proceedings of the AAAI Symposium on Quantum Interaction, Stanford, 26-28 March 2007, 9597.

[30] Busemeyer, J.R., Wang, Z. and Lambert-Mogiliansky, A. (2009) Empirical Comparison of Markov and Quantum Models of Decision Making. Journal of Mathematical Psychology, 53, 423-433. http://dx.doi.org/10.1016/j.jmp.2009.03.002

[31] Chalkins, S.D. and Bell, M.A. (2010) Child Development at the Intersection of Emotion and Cognition. http://www.apa.org/pubs/books/4318067.aspx 